\title{
On the Polyconvolution with the Weight Function for the Fourier Cosine, Fourier Sine, and the Kontorovich-Lebedev Integral Transforms
}

\author{
Nguyen Xuan Thao \\ Faculty of Applied Mathematics and Informatics, Hanoi University of Technology, \\ No. 1, Dai Co Viet, Hanoi, Vietnam \\ Correspondence should be addressed to Nguyen Xuan Thao, thaonxbmai@yahoo.com \\ Received 26 December 2009; Revised 5 April 2010; Accepted 17 May 2010 \\ Academic Editor: Slimane Adjerid
}

Copyright (c) 2010 Nguyen Xuan Thao. This is an open access article distributed under the Creative Commons Attribution License, which permits unrestricted use, distribution, and reproduction in any medium, provided the original work is properly cited.

The polyconvolution with the weight function $\gamma$ of three functions $f, g$, and $h$ for the integral transforms Fourier sine $\left(F_{s}\right)$, Fourier cosine $\left(F_{c}\right)$, and Kontorovich-Lebedev $\left(K_{i y}\right)$, which is denoted by $\stackrel{r}{*}(f, g, h)(x)$, has been constructed. This polyconvolution satisfies the following factorization property $F_{C}(*(f, g, h))(y)=\sin y\left(F_{s} f\right)(y) \cdot\left(F_{c} g\right)(y) \cdot\left(K_{i y} h\right)(y)$, for all $y>0$. The relation of this polyconvolution to the Fourier convolution and the Fourier cosine convolution has been obtained. Also, the relations between the polyconvolution product and others convolution product have been established. In application, we consider a class of integral equations with Toeplitz plus Hankel kernel whose solution in closed form can be obtained with the help of the new polyconvolution. An application on solving systems of integral equations is also obtained.

\section{Introduction}

The convolution of two functions $f$ and $g$ for the Fourier transform is well known [1]

$$
\left(\begin{array}{c}
f * g \\
F
\end{array}\right)(x)=\frac{1}{\sqrt{2 \pi}} \int_{-\infty}^{\infty} f(x-y) g(y) d y, \quad x \in \mathbb{R}
$$

This convolution has the factorization equality as below

$$
F(f \underset{F}{*} g)(y)=(F f)(y)(F g)(y), \quad \forall y \in \mathbb{R}
$$


where $F$ denotes the Fourier transform

$$
(F f)(y)=\frac{1}{\sqrt{2 \pi}} \int_{-\infty}^{\infty} f(x) e^{-i x y} d x
$$

The convolution of $f$ and $g$ for the Kontorovich-Lebedev integral transform has been studied in [2]

$$
(f \underset{K-L}{*} g)(x)=\frac{1}{2 x} \iint_{0}^{\infty} \exp \left[-\frac{1}{2}\left(\frac{x u}{v}+\frac{x v}{u}+\frac{u v}{x}\right)\right] f(u) g(v) d u d v, \quad x>0
$$

for which the following factorization identity holds:

$$
K_{i y}(f \underset{K-L}{*} g)=\left(K_{i y} f\right) \cdot\left(K_{i y} g\right), \quad \forall y>0
$$

Here $K_{i y}$ is the Kontorovich-Lebedev transform [3]

$$
K_{i x}[f]=\int_{0}^{\infty} K_{i x}(t) f(t) d t
$$

and $K_{i x}(t)$ is the Macdonald function [4].

The convolution of two functions $f$ and $g$ for the Fourier cosine is of the form [1]

$$
(f * g)(x)=\frac{1}{\sqrt{2 \pi}} \int_{0}^{\infty} f(y)[g(|x-y|)+g(x+y)] d y, \quad x>0
$$

which satisfied the following factorization equality:

$$
F_{c}\left(\begin{array}{c}
f * g \\
1
\end{array}\right)(y)=\left(F_{c} f\right)(y)\left(F_{c} g\right)(y), \quad \forall y>0
$$

Here the Fourier cosine transform is of the form

$$
\left(F_{c} f\right)(y)=\sqrt{\frac{2}{\pi}} \int_{0}^{\infty} \cos y x \cdot f(x) d x, \quad y>0 .
$$

The convolution with a weight function $\gamma(x)=\sin x$ of two functions $f$ and $g$ for the Fourier sine transform has been introduced in $[5,6]$

$$
\begin{array}{r}
(f \stackrel{r}{f} g)(x)=\frac{1}{2 \sqrt{2 \pi}} \int_{0}^{+\infty} f(y)[\operatorname{sign}(x+y-1) g(|x+y-1|)+\operatorname{sign}(x-y+1) g(|x-y+1|) \\
-g(x+y+1)-\operatorname{sign}(x-y-1) g(|x-y-1|)] d y, \quad x>0
\end{array}
$$


and the following factorization identity holds:

$$
F_{s}(f * g)(y)=\sin y\left(F_{s} f\right)(y)\left(F_{s} g\right)(y), \quad \forall y>0
$$

Here the Fourier sine is of the form

$$
\left(F_{s} f\right)(y)=\sqrt{\frac{2}{\pi}} \int_{0}^{\infty} \sin y x \cdot f(x) d x, \quad y>0 .
$$

The generalized convolution of two functions $f$ and $g$ for the Fourier sine and Fourier cosine transforms has been studied in [1]

$$
(f \underset{2}{*} g)(x)=\frac{1}{\sqrt{2 \pi}} \int_{0}^{\infty} f(u)[g(|x-u|)-g(x+u)] d u, \quad x>0
$$

and proved the following factorization identity [1]:

$$
F_{S}\left(\begin{array}{l}
f * g \\
2
\end{array}\right)(y)=\left(F_{s} f\right)(y) \cdot\left(F_{c} g\right)(y), \quad \forall y>0
$$

The generalized convolution of two functions $f$ and $g$ for the Fourier cosine and the Fourier sine transforms is defined by [7]

$$
(f * g)(x)=\frac{1}{\sqrt{2 \pi}} \int_{0}^{\infty} f(u)[\operatorname{sign}(u-x) g(|u-x|)+g(u+x)] d u, \quad x>0 .
$$

For this generalized convolution, the following factorization equality holds:

$$
F_{c}(f * g)(y)=\left(F_{s} f\right)(y)\left(F_{s} g\right)(y), \quad \forall y>0
$$

The generalized convolution with the weight function $\gamma(x)=\sin x$ for the Fourier cosine and the Fourier sine transforms of $f$ and $g$ has been introduced in [8]

$$
\begin{aligned}
\left(\begin{array}{r}
f \\
\underset{1}{*} g
\end{array}\right)(x)=\frac{1}{2 \sqrt{2 \pi}} \int_{0}^{\infty} f(u)[g(|x+u-1|)+g(|x-u+1|) & \\
& -g(x+u+1)-g(|x-u-1|)] d u, \quad x>0 .
\end{aligned}
$$

It satisfies the factorization property

$$
F_{c}\left(\begin{array}{r}
f \underset{*}{*} g \\
{ }_{1}
\end{array}\right)(y)=\sin y\left(F_{s} f\right)(y)\left(F_{c} g\right)(y), \quad \forall y>0
$$


The generalized convolution with the weight function $\gamma(x)=\sin x$ of $f$ and $g$ for the Fourier sine and Fourier cosine has been studied in [9]

$$
\begin{aligned}
\left(\begin{array}{r}
f \\
\underset{2}{*}
\end{array}\right)(x)=\frac{1}{2 \sqrt{2 \pi}} \int_{0}^{\infty} f(u)[g(|x+u-1|)+g(|x-u-1|) & \\
& -g(x+u+1)-g(|x-u+1|)] d u, \quad x>0,
\end{aligned}
$$

and satisfies the following factorization identity:

$$
F_{s}(\underset{2}{f} \underset{2}{r} g)(y)=\sin y\left(F_{c} f\right)(y)\left(F_{c} g\right)(y), \quad \forall y>0
$$

Recently, the following generalized convolutions for Fourier cosine, Kontorovich-Lebedev and Fourier sine, Kontorovich-Lebedev are studied in [10] (f. 21)

$$
(f * g)_{\left\{c_{S}\right\}}(x)=\frac{1}{2 \pi x} \int_{\mathbb{R}_{+}^{2}} f(u) g(v)\left[e^{-x \cosh (u-v)} \pm e^{-x \cosh (u+v)}\right] d u d v, \quad x>0 .
$$

The respective factorization equalities are [10]

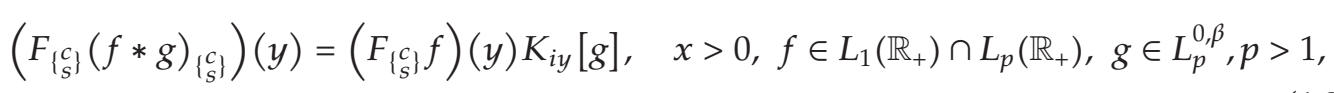

where

$$
L_{p}^{0, \beta}=\left\{f: \int_{0}^{\infty}|f(t)|^{p} K_{0}(\beta t) d t<\infty, 0<\beta \leqslant 1\right\}
$$

In 1997, Kakichev introduced a constructive method for defining a polyconvolution with a weight function $\gamma$ of functions $f_{1}, f_{2}, \ldots, f_{n}$ for the integral transforms $K, K_{1}, K_{2}, \ldots, K_{n}$, which are denoted by ${ }^{r}\left(f_{1}, f_{2}, \ldots, f_{n}\right)(x)$, such that the following factorization property holds [11]:

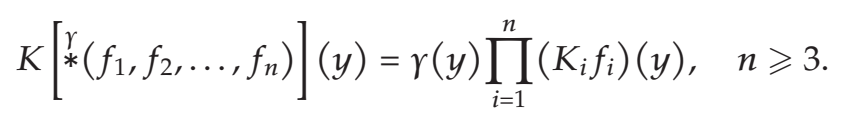

Polyconvolutions for the Hilbert, Stieltjes, Fourier cosine, and Fourier sine integral transforms have been studied in [12].

The polyconvolution of $f, g$, and $h$ for the Fourier cosine and the Fourier sine transforms has the form [13]

$$
\begin{aligned}
*(f, g, h)(x)=\frac{1}{2 \pi} \iint_{0}^{\infty} f(u) g(v)[h(|x+u-v|)+h(x-u+v \mid) & \\
& -h(|x-u-v|)-h(x+u+v)] d u d v, \quad x>0,
\end{aligned}
$$


which satisfies the following factorization property:

$$
F_{c}(*(f, g, h))(y)=\left(F_{s} f\right)(y) \cdot\left(F_{s} g\right)(y) \cdot\left(F_{c} h\right)(y), \quad \forall y>0
$$

In recent years, many sciences were interested in the theory of convolution for the integral transforms and gave several interesting application (see $[3,14-21]$ ), specially, the integral equations with the Toeplitz plus Hankel kernel [22-24]

$$
f(x)+\int_{0}^{\infty}\left[k_{1}(x+y)+k_{2}(x-y)\right] f(y) d y=g(x), \quad x>0,
$$

where $k_{1}, k_{2}$, and $g$ are known functions, and $f$ is an unknown function. Many partial cases of this equation can be solved in closed form with the help of the convolutions and generalized convolutions. In this paper, we construct and investigate the polyconvolution for the Fourier sine, Fourier cosine, and the Kontorovich-Lebedev transforms. Several properties of this new polyconvolution and its application on solving integral equation with Toeplitz plus Hankel equation and systems of integral equations are obtained.

\section{Polyconvolution}

Definition 2.1. The polyconvolution with the weight function $\gamma=\sin x$ of functions $f, g$, and $h$ for the Fourier cosine, Fourier sine, and the Kontorovich-Lebedev integral transforms is defined as follows:

$$
\stackrel{r}{*}(f, g, h)(x)=\iiint_{0}^{\infty} \theta(x, u, v, w) f(u) g(v) h(w) d u d v d w
$$

where

$$
\begin{aligned}
\theta(x, u, v, w)=\frac{1}{4 \sqrt{2 \pi}} & {\left[e^{-w \cosh (x+v+u-1)}+e^{-w \cosh (x+v-u+1)}+e^{-w \cosh (x-v+u-1)}+e^{-w \cosh (x-v-u+1)}\right.} \\
& \left.-e^{-w \cosh (x+v+u+1)}-e^{-w \cosh (x+v-u-1)}-e^{-w \cosh (x-v+u+1)}-e^{-w \cosh (x-v-u-1)}\right] .
\end{aligned}
$$

Theorem 2.2. Let $f$ and $g$ be functions in $L_{1}\left(\mathbb{R}_{+}\right)$, and let $h$ be a function in $L_{1}\left(1 / \sqrt{w}, \mathbb{R}_{+}\right)$; then the polyconvolution (2.1) belongs to $L_{1}\left(\mathbb{R}_{+}\right)$and satisfies the following factorization equality:

$$
F_{c}(\stackrel{r}{*}(f, g, h))(y)=\sin y\left(F_{s} f\right)(y) \cdot\left(F_{c} g\right)(y) \cdot\left(K_{i y} h\right), \quad \forall y>0 .
$$


Proof. Since $\left|e^{-w \cosh (x+u+v-1)}-e^{-w \cosh (x+u+v-1)}\right| \leqslant 1 / \sqrt{w}$ for sufficient large $w>0$, we have

$$
\begin{aligned}
|r(f, g, h)(x)| & \leqslant \frac{1}{4 \sqrt{2 \pi}} \iiint_{0}^{\infty}|f(u)||g(v)||h(w)||\theta(x, u, v, w)| d u d v d w \\
& \leqslant \frac{1}{\sqrt{2 \pi}} \int_{0}^{\infty}|f(u)| d u \cdot \int_{0}^{\infty}|g(v)| d v \cdot \int_{0}^{\infty} \frac{1}{\sqrt{w}}|h(w)| d w<+\infty .
\end{aligned}
$$

On the other hand, note that $\cosh (x+u+v-1) \geqslant(x+u+v-1)^{2} / 2$; we have

$$
e^{-w \cosh (x+u+v-1)} \leqslant e^{-w\left((x+u+v-1)^{2} / 2\right)}, \quad \forall w>0 .
$$

Using formula 3.321.3, page 321, in [4], we have

$$
\begin{aligned}
\int_{0}^{\infty} e^{-w \cosh (x+u+v-1)} d x & \leqslant \sqrt{\frac{2}{w}} \int_{0}^{\infty} e^{-(\sqrt{w / 2}(x+u+v-1))^{2}} d\left(\sqrt{\frac{w}{2}}(x+u+v-1)\right) \\
& \leqslant 2 \sqrt{\frac{2}{w}} \int_{0}^{\infty} e^{-s^{2}} d s=\sqrt{\frac{2 \pi}{w}}
\end{aligned}
$$

It shows that

$$
\begin{aligned}
& \iiint \int_{0}^{\infty} e^{-w \cosh (x+u+v-1)}|f(u)||g(v)||h(w)| d u d v d w d x \\
& \quad \leqslant \iiint_{0}^{\infty} \sqrt{\frac{2 \pi}{w}}|h(w)||f(u)||g(v)| d u d v d w \\
& \quad \leqslant \sqrt{2 \pi} \int_{0}^{\infty} \frac{1}{\sqrt{w}}|h(w)| d w \cdot \int_{0}^{\infty}|f(u)| d u \cdot \int_{0}^{\infty}|g(v)| d v<+\infty .
\end{aligned}
$$

By the same way, we obtain similar estimations for the 7 other terms. Therefore, from formulas (2.1), (2.2), and (2.7), we have

$$
\int_{0}^{\infty}|r(f, g, h)(x)| d x<+\infty
$$

It shows that the polyconvolution (2.1) belongs to $L_{1}\left(\mathbb{R}_{+}\right)$. We now prove the factorization equality (2.3). Indeed, we have

$$
\begin{aligned}
& \sin y\left(F_{s} f\right)(y)\left(F_{c} g\right)(y)\left(K_{i y} h\right) \\
& \quad=\frac{2}{\pi} \iiint_{0}^{\infty} \sin y \sin (y u) \cos (y v) K_{i y}(w) f(u) g(v) h(w) d u d v d w .
\end{aligned}
$$


Using formula 2, page 130 in [4], we get

$$
\begin{aligned}
& \sin y\left(F_{S} f\right)(y)\left(F_{c} g\right)(y)\left(K_{i y} h\right) \\
& =\frac{2}{\pi} \iiint \int_{0}^{\infty} \sin y \sin (y u) \cos (y v) \cos (y \alpha) e^{-w \cosh \alpha} f(u) g(v) h(w) d u d v d w d \alpha \\
& =\frac{1}{4 \pi} \iiint \int_{0}^{\infty} e^{-w \cosh \alpha}[\cos y(u-1+v+\alpha)+\cos y(u-1-v-\alpha)+\cos y(u-1+v-\alpha) \\
& +\cos y(u-1-v+\alpha)-\cos y(u+1+v+\alpha)-\cos y(u+1-v-\alpha) \\
& -\cos y(u+1+v-\alpha)-\cos y(u+1-v+\alpha)] \\
& \times f(u) g(v) h(w) d u d v d w d \alpha .
\end{aligned}
$$

Interchanging variables, we have

$$
\begin{array}{r}
\int_{0}^{\infty} e^{-w \cosh \alpha}[\cos y(u-1+v+\alpha)-\cos y(u+1+v+\alpha)] d \alpha \\
=\int_{0}^{\infty} \cos y x\left[e^{-w \cosh (x-u+1-v)}-e^{-w \cosh (x-u-1-v)}\right] d x
\end{array}
$$

Similarly,

$$
\begin{array}{r}
\int_{0}^{\infty} e^{-w \cosh \alpha}[\cos y(u-1-v+\alpha)-\cos y(u+1-v+\alpha)] d \alpha \\
\quad \int_{0}^{\infty} \cos y x\left[e^{-w \cosh (x-u+1+v)}-e^{-w \cosh (x-u-1+v)}\right] d x \\
\int_{0}^{\infty} e^{-w \cosh \alpha}[\cos y(u-1-v-\alpha)-\cos y(u+1-v-\alpha)] d \alpha \\
=\int_{0}^{\infty} \cos y x\left[e^{-w \cosh (x+u-1-v)}-e^{-w \cosh (x+u+1-v)}\right] d x \\
\int_{0}^{\infty} e^{-w \cosh \alpha}[\cos y(u-1+v-\alpha)-\cos y(u+1+v-\alpha)] d \alpha \\
=\int_{0}^{\infty} \cos y x\left[e^{-w \cosh (x+u-1+v)}-e^{-w \cosh (x+u+1+v)}\right] d x .
\end{array}
$$

From fomulae (2.10)-( 2.8), we have

$$
\sin y\left(F_{s} f\right)(y)\left(F_{c} g\right)(y)\left(K_{i y} h\right)=F_{c}(\stackrel{r}{*}(f, g, h))(y) .
$$

The proof is complete. 
Definition 2.3. Let $f$ be a function in $L_{1}\left(\mathbb{R}_{+}\right)$and let $h$ be a function in $L_{1}\left(\beta, \mathbb{R}_{+}\right)$; their norms are defined as follows:

$$
\|f\|_{L_{1}\left(\mathbb{R}_{+}\right)}=\int_{0}^{\infty}|f(x)| d x, \quad\|h\|_{L_{1}\left(\beta, \mathbb{R}_{+}\right)}=\int_{0}^{\infty} \beta(v)|h(v)| d v .
$$

here $\beta(v)=2 / \sqrt{v}$.

Theorem 2.4. Let $f$ and $g$ be functions in $L_{1}\left(\mathbb{R}_{+}\right)$, and let $h$ be function in $L_{1}\left(\beta, \mathbb{R}_{+}\right)$; then the following estimation holds:

$$
\|*(f, g, h)\|_{L_{1}\left(\mathbb{R}_{+}\right)} \leqslant\|f\|_{L_{1}\left(\mathbb{R}_{+}\right)}\|g\|_{L_{1}\left(\mathbb{R}_{+}\right)}\|h\|_{L_{1}\left(\beta, \mathbb{R}_{+}\right)}
$$

Proof. From formulas (2.1), (2.2), and (2.7), we have

$$
\int|*(f, g, h)(x)| d x \leqslant 2 \int_{0}^{\infty} \frac{1}{\sqrt{w}}|h(w)| d w \cdot \int_{0}^{\infty}|f(u)| d u \cdot \int_{0}^{\infty}|g(v)| d v .
$$

Therefore, by Definition 2.3,

$$
\|*(f, g, h)\|_{L_{1}\left(\mathbb{R}_{+}\right)} \leqslant\|f\|_{L_{1}\left(\mathbb{R}_{+}\right)}\|g\|_{L_{1}\left(\mathbb{R}_{+}\right)}\|h\|_{L_{1}\left(\beta, \mathbb{R}_{+}\right)}
$$

Proposition 2.5. Let $f, g \in L_{1}\left(\mathbb{R}_{+}\right)$, and let $h \in L_{1}\left(1 / \sqrt{w}, \mathbb{R}_{+}\right)$; then the following identity holds:

$$
\begin{aligned}
\stackrel{r}{*}(f, g, h)=\frac{1}{2} \sqrt{\frac{\pi}{2}} \int_{0}^{\infty} h(w) & {\left[\left(\left(g * e^{-w \cosh t}\right){ }_{F}^{*}(f(|t|) \operatorname{sign} t)\right)(x+1)\right.} \\
& \left.-\left(\left(g * e^{-w \cosh t}\right){ }_{F}^{*}(f(|t|) \operatorname{sign} t)\right)(x-1)\right] d w .
\end{aligned}
$$

Proof. From the definition (2.1) of the polyconvolution and the convolution (1.7), we have

$$
\begin{aligned}
\stackrel{\gamma}{*}( & f, g, h)(x) \\
=\frac{1}{4} \iint_{0}^{\infty} f(u) h(w)[ & \left(g * e^{-w \cosh t}\right)(x-u+1)+\left(g * e^{-w \cosh t}\right)(x+u-1) \\
& \left.-\left(g * e^{-w \cosh t}\right)(x+u+1)-\left(g * e^{-w \cosh t}\right)(x-u-1)\right] d u d w .
\end{aligned}
$$


From (2.19) and calculation, we obtain

$$
\begin{aligned}
\underset{1}{*}(f, g, h)(x)=\sqrt{\frac{\pi}{2}} \int_{0}^{\infty} h(w) & {\left[\left(\left(g * e^{-w \cosh t}\right){ }_{F}^{*} f(|t|) \operatorname{sign} t\right)(x+1)\right.} \\
- & \left.\left(\left(g * e^{-w \cosh t}\right){ }_{F}^{*} f(|t|) \operatorname{sign} t\right)(x-1)\right] d w .
\end{aligned}
$$

The proof is complete.

Theorem 2.6. Let $f, g, h$ be functions in $L_{1}\left(\mathbb{R}_{+}\right), \gamma(x)=\sin x$, and let $l$ and $k$ be functions in $L(1 / \sqrt{w}, \mathbb{R})$; then the following properties holds:
(a) $\stackrel{r}{*}(f, \stackrel{r}{r}(g, h, k), l)=\stackrel{r}{*}(\underset{g}{g}, \stackrel{r}{*}(f, h, k), l)$;
(b) $\stackrel{r}{*}(f \underset{2}{*} g, h, k)=\stackrel{r}{*}(f, g * h, k)$;
(c) $\stackrel{r}{*}(f * \underset{*}{*} g, h, k)=\stackrel{r}{*}(f, \underset{1}{g} \stackrel{r}{*} h, k)$;
(d) $*(f \underset{2}{r} \underset{*}{*} g, h, k)=\stackrel{r}{*}(f \underset{2}{*} h, g, g)$;
(e) $\stackrel{r}{*}(f, g \underset{3}{*} h, k)=\stackrel{r}{*}(g, f \underset{3}{*} h, k)$.

Proof. First, we prove the assertion (c). From Theorem 2.2 and the convolutions (1.17), (1.10), we have

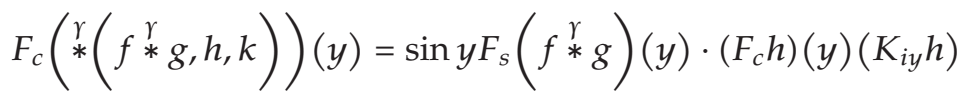

$$
\begin{aligned}
& =\sin y \sin y\left(F_{s} f\right)(y)\left(F_{s} g\right)(y)\left(F_{c} h\right)(y)\left(K_{i y} h\right) \\
& =\sin y \cdot\left(F_{s} f\right)(y) \cdot F_{c}(\underset{g}{*} \stackrel{r}{*} h)(y) \cdot\left(K_{i y} h\right) \\
& =F_{c}(\stackrel{r}{*}(f, \underset{1}{\stackrel{\gamma}{*} h, k})) .
\end{aligned}
$$

Therefore, the part (c) holds. Other parts can be proved in a similar way.

\section{Applications in Solving Integral Equations and Systems of Integral Equations}

Consider the integral equation

$$
\begin{aligned}
f(x)+\iiint_{0}^{\infty} \theta(x, u, v, w) g(u) f(v) h(w) d u d v d w \\
\quad+\int_{0}^{\infty} \theta_{1}(x, u) f(u) d u+\int_{0}^{\infty} \theta_{2}(x, u) f(u) d u=\varphi(x), \quad x>0,
\end{aligned}
$$


where $g, h, k, l$, and $\varphi$ are known functions, $f$ is an unknown function, $\theta(x, u, v, w)$ is given by the formula (2.2), and

$$
\begin{aligned}
& \theta_{1}(x, u)=\frac{1}{2 \sqrt{2 \pi}}[k(x+u+1)-k(|x+u-1|) \operatorname{sign}(x+u-1) \\
& \quad+k(|x-u+1|) \operatorname{sign}(x-u+1)-k(|x-u-1|) \operatorname{sign}(x-u-1)], \\
& \theta_{2}(x, u)=\frac{1}{\sqrt{2 \pi}}[l(x+u)+l(|x-u|)] .
\end{aligned}
$$

Theorem 3.1. Suppose that $g, l, \varphi, k_{1}, k_{2} \in L_{1}\left(\mathbb{R}_{+}\right), h \in L_{1}\left(1 / \sqrt{v}, \mathbb{R}_{+}\right), k=k_{1}{\underset{2}{2}}_{2}$ such that

$$
1+\sin y\left(F_{s} g\right)(y)\left(K_{i y} h\right)+\left(F_{s} k_{1}\right)(y)\left(F_{c} k_{2}\right)(y)+\left(F_{c} l\right)(y) \neq 0,
$$

then (3.1) has a unique solution in $L_{1}\left(\mathbb{R}_{+}\right)$whose closed form is

$$
f(x)=\varphi(x)-(\varphi * \xi)(x)
$$

Here $\xi \in L_{1}\left(\mathbb{R}_{+}\right)$is defined uniquely by

$$
\left(F_{c} \xi\right)(y)=\frac{\sin y\left(F_{s} g\right)(y)\left(K_{i y} h\right)+\sin y\left(F_{s} k_{1}\right)(y)\left(F_{c} k_{2}\right)(y)+\left(F_{c} l\right)(y)}{1+\sin y\left(F_{s} g\right)(y)\left(K_{i y} h\right)+\sin y\left(F_{s} k_{1}\right)(y)\left(F_{c} k_{2}\right)(y)+\left(F_{c} l\right)(y)}
$$

Proof. We obtain the following lemmas.

Lemma 3.2. For $f, k \in L_{1}\left(\mathbb{R}_{+}\right)$, then the following operator also belongs to $L_{1}\left(\mathbb{R}_{+}\right)$

$$
\int_{0}^{\infty} f(u) \theta_{1}(x, u) d u
$$

Moreover, the following factorization equality holds:

$$
F_{c}\left(\int_{0}^{\infty} f(u) \theta_{1}(x, u) d u\right)(y)=\sin y \cdot\left(F_{s} k\right)(y)\left(F_{c} f\right)(y), \quad \forall y>0 .
$$

Lemma 3.3. Let $g \in L_{1}\left(\mathbb{R}_{+}\right), h \in L_{1}\left(1 / \sqrt{v}, \mathbb{R}_{+}\right)$; then the generalized convolution $\underset{3}{g * h}(x)$ belongs to $L_{1}\left(\mathbb{R}_{+}\right)$and the respectively factorization equality is

$$
F_{c}(\underset{3}{g * h})(y)=\sin y\left(F_{s} g\right)(y)\left(K_{i y} h\right), \quad \forall y>0
$$


where

$$
\begin{aligned}
& (\underset{3}{\underset{3}{*} h})(x)=\frac{1}{4} \iint_{0}^{\infty}\left[e^{-v \cosh (x+u-1)}+e^{-v \cosh (x-u+1)}-e^{-v \cosh (x+u+1)}-e^{-v \cosh (x-u-1)}\right] \\
& \times g(u) h(v) d u d v, \quad x>0 .
\end{aligned}
$$
have

We now prove Theorem 3.1 with the help of convolution (1.7), Lemmas 1, and 2. We

$$
\begin{aligned}
& \left(F_{c} f\right)(y)+\sin y\left(F_{s} g\right)(y) \cdot\left(F_{c} f\right)(y) \cdot\left(K_{i y} h\right) \\
& \quad+\left(F_{s} k\right)(y) \cdot\left(F_{c} f\right)(y) \sin y+\left(F_{c} l\right)(y) \cdot\left(F_{c} f\right)(y)=\left(F_{c} \varphi\right)(y) .
\end{aligned}
$$

Therefore, by the given condition,

$$
\left(F_{c} f\right)(y)=\left(F_{c} \varphi\right)(y)\left(1-\frac{\sin y\left(F_{s} g\right)(y)\left(K_{i y} h\right)+\sin y\left(F_{s} k\right)(y)+\left(F_{c} l\right)(y)}{1+\sin y\left(F_{s} g\right)(y)\left(K_{i y} h\right)+\sin y\left(F_{s} k\right)(y)+\left(F_{c} l\right)(y)}\right)
$$

By the hypothesis $k=k_{1} \underset{2}{*} k_{2}$, we see that $\sin y\left(F_{s} k\right)(y)=F_{c}\left(k_{1} \underset{1}{r} k_{2}\right)(y)$; using Lemma 3.3, we get

$$
\left(F_{c} f\right)(y)=\left(F_{c} \varphi\right)(y)\left(1-\frac{F_{c}(\underset{8}{\stackrel{r}{*} h})(y)+F_{c}\left(\underset{1}{k_{1} \stackrel{r}{*} k_{2}}\right)(y)+\left(F_{c} l\right)(y)}{1+F_{c}(\underset{4}{g} \underset{*}{*} h)(y)+F_{c}\left(\underset{1}{k_{1}^{*} k_{2}}\right)(y)+\left(F_{c} l\right)(y)}\right) .
$$

In virtue of the Wiener-Levy theorem [25], by the given condition, there exists a function $\xi \in L_{1}\left(\mathbb{R}_{+}\right)$such that

$$
\left(F_{c} \xi\right)(y)=\frac{\sin y\left(F_{s} g\right)(y)\left(K_{i y} h\right)+\sin y\left(F_{s} k_{1}\right)(y)\left(F_{c} k_{2}\right)(y)+\left(F_{c} l\right)(y)}{1+\sin y\left(F_{s} g\right)(y)\left(K_{i y} h\right)+\sin y\left(F_{s} k_{1}\right)(y)\left(F_{c} k_{2}\right)(y)+\left(F_{c} l\right)(y)} .
$$

From (3.12) and (3.13), we have

$$
\left(F_{c} f\right)(y)=\left(F_{c} \varphi\right)(y)\left[1-\left(F_{c} \xi\right)(y)\right]
$$

Then the solution in $L_{1}\left(\mathbb{R}_{+}\right)$of (3.1) has the form

$$
f(x)=\varphi(x)-\left(\begin{array}{c}
\varphi * \xi \\
1
\end{array}\right)(x) .
$$

The proof is complete. 
Remark 3.4. The integral equation (3.1) is a special case of the integral equation with the Toeplitz plus Hankel kernel (1.27) for $x>0$ and

$$
\begin{aligned}
& k_{1}(t) \\
& =\frac{1}{2 \sqrt{2 \pi}}[k(t+1)-k(|t-1|) \operatorname{sign}(t-1)]+\frac{1}{\sqrt{2 \pi}} l(t) \\
& \quad+\frac{1}{4 \sqrt{2 \pi}} \iint_{0}^{\infty} g(u) h(w)\left[e^{-w \cosh (t+u-1)}+e^{-w \cosh (t-u+1)}-e^{-w \cosh (t+u+1)}-e^{-w \cosh (t-u-1)}\right] d u d w \\
& k_{2}(t) \\
& =\frac{1}{2 \sqrt{2 \pi}}[k(|t+1|) \operatorname{sign}(t+1)-k(|t-1|) \operatorname{sign}(t-1)]+\frac{1}{\sqrt{2 \pi}} l(|t|) \\
& \quad+\frac{1}{4 \sqrt{2 \pi}} \iint_{0}^{\infty} g(u) h(w)\left[e^{-w \cosh (t+u-1)}+e^{-w \cosh (t-u+1)}-e^{-w \cosh (t+u+1)}-e^{-w \cosh (t-u-1)}\right] d u d w .
\end{aligned}
$$

Next, we consider the following system of two integral equations:

$$
\begin{array}{r}
f(x)+\iiint_{0}^{\infty} \theta(x, u, v, w) g(u) h(v) k(w) d u d v d w+\int_{0}^{\infty} \theta_{3}(x, u) g(u) d u=p(x) \\
\int_{0}^{\infty} \theta_{4}(x, u) f(u) d u+\int_{0}^{\infty} \theta_{5}(x, u) f(u) d u+g(x)=q(x) .
\end{array}
$$

Here $\theta(x, u, v, w)$ is defined by (2.2), and

$$
\begin{aligned}
& \theta_{3}(x, u)=\frac{1}{\sqrt{2 \pi}}[l(x+u)-l(|x-u|) \operatorname{sign}(x-u)] \\
& \theta_{4}(x, u)=\frac{1}{\sqrt{2 \pi}}[\xi(x+u)+\xi(|x-u|) \operatorname{sign}(x-u)] \\
& \theta_{5}(x, u)=\frac{1}{2 \sqrt{2 \pi}}[\eta(\mid x+u-1)+\eta(|x-u-1|)-\eta(x+u+1)-\eta(x-u+1)]
\end{aligned}
$$

$h, k, l, \xi, \eta, p, q$ are known functions, and $f$ and $g$ are unknown functions.

Theorem 3.5. Given that $p, q, h, l, \xi, \eta_{1}, \eta_{2} \in L_{1}\left(\mathbb{R}_{+}\right)$and $k \in L_{1}\left(\beta, \mathbb{R}_{+}\right), \eta=\eta_{1}^{*} \eta_{3}$ such that $1-\left(F_{c} \psi\right)(y) \neq 0$, where

$$
\psi(x)=(\stackrel{r}{*}(\xi, h, k))(x)-(\xi * l)(x)-*\left(\underset{3}{\eta_{1} * \eta_{2}}, h, k\right)(x)-\left(\begin{array}{c}
l * \eta \\
1
\end{array}\right)(x) .
$$


Then the system (3.17) has a unique solution in $L_{1}\left(\mathbb{R}_{+}\right) \times L_{1}\left(\mathbb{R}_{+}\right)$whose closed form is as follows

$$
\begin{aligned}
& f(x)=p(x)-(*(q, h, k))(x)+(q * l)+\left(\begin{array}{c}
l * p \\
1
\end{array}\right)(x) \\
& +\left(\begin{array}{l}
r \\
*(q, h, k) \\
1
\end{array}\right)(x)+((q \underset{3}{*} l) * l)(x)
\end{aligned}
$$

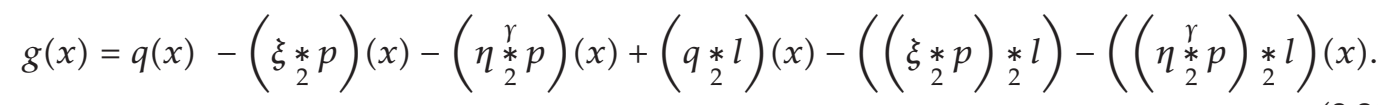

Here, $l \in L_{1}\left(\mathbb{R}_{+}\right)$is defined by

$$
\left(F_{c} l\right)(y)=\frac{\left(F_{c} \psi\right)(y)}{1-\left(F_{c} \psi\right)(y)}
$$

Proof. We need the following lemma.

Lemma 3.6. Let $\xi, f \in L_{1}\left(\mathbb{R}_{+}\right)$; then

$$
\begin{gathered}
\int_{0}^{\infty}[\xi(x+u)+\xi(|x-u|) \operatorname{sign}(x-u)] f(u) d u \in L_{1}\left(\mathbb{R}_{+}\right) \\
F_{s}\left(\frac{1}{\sqrt{2 \pi}} \int_{0}^{\infty}[\xi(x+u)+\xi(|x-u|) \operatorname{sign}(x-u)] f(u) d u\right)(y)=\left(F_{s} \xi\right)(y)\left(F_{c} f\right)(y), \quad \forall y>0 .
\end{gathered}
$$
have

Using Theorem 2.2, Lemma 3.6, and the generalized convolution (1.15), (1.19), we

$$
\begin{gathered}
\left(F_{c} f\right)(y)+\sin y\left(F_{s} g\right)(y)\left(F_{c} h\right)(y)\left(K_{i y} k\right)+\left(F_{s} l\right)(y)\left(F_{s} g\right)(y)=\left(F_{c} p\right)(y), \\
\left(F_{s} \xi\right)(y)\left(F_{c} f\right)(y)+\sin y\left(F_{c} \eta\right)(y)\left(F_{c} f\right)(y)+\left(F_{s} g\right)(y)=\left(F_{s} q\right)(y) .
\end{gathered}
$$

On the other hand, from $\eta=\eta_{1} * \eta_{2}$ we have $\sin y\left(F_{c} \eta\right)(y)=F_{s}\left(\eta_{1} * \eta_{2}\right)(y)$. Therefore, using Theorem 2.2 and the generalized convolution (1.15), (1.17), we have

$$
\begin{aligned}
& \Delta=\left|\begin{array}{cc}
1 & \sin y\left(F_{c} h\right)(y) \cdot\left(K_{i y} k\right)+\left(F_{s} l\right)(y) \\
\left(F_{s} \xi\right)(y)+\sin y\left(F_{c} \eta\right)(y) & 1
\end{array}\right|
\end{aligned}
$$

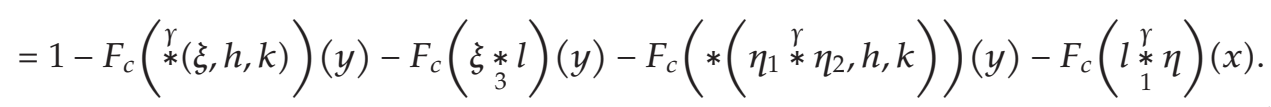


Hence, in view of the Wiener-Levy theorem [25], by the given condition, there is a unique function $l \in L_{1}\left(\mathbb{R}_{+}\right)$such that

$$
\frac{1}{\Delta}=1+\left(F_{c} l\right)(y)
$$

where

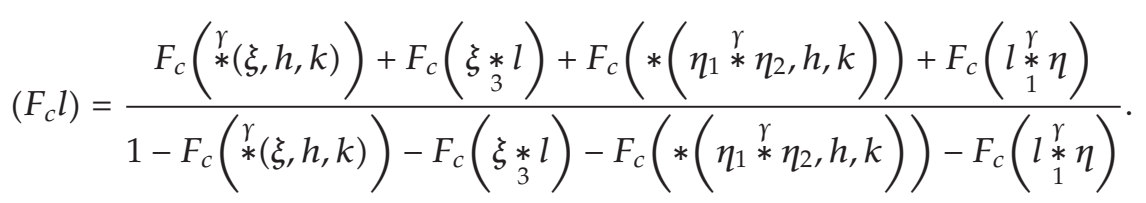

On the other hand, using Theorem 2.2 and the generalized convolution (1.15), we have

$$
\begin{aligned}
\Delta_{1} & =\left|\begin{array}{cc}
\left(F_{c} p\right)(y) & \sin y\left(F_{c} h\right)(y) \cdot\left(K_{i y} k\right)+\left(F_{s} l\right)(y) \\
\left(F_{s} q\right)(y) & 1
\end{array}\right| \\
& =\left(F_{c} p\right)(y)-F_{c}(*(q, h, k))(y)-F_{c}(\underset{3}{*} l)(y) .
\end{aligned}
$$

Hence, from (3.25), (3.27) we have

$$
\begin{aligned}
\left(F_{s} f\right)(y)= & {\left[1+\left(F_{c} l\right)(y)\right]\left[\left(F_{c} p\right)(y)-F_{c}(*(q, h, k))(y)-F_{c}\left(q_{3}^{* l}\right)(y)\right] } \\
= & \left(F_{c} p\right)(y)-F_{c}(*(q, h, k))-F_{c}(q * l)+F_{c}\left(l_{1}^{*} p\right)(y)-F_{c}(*(q, h, k) * l)(y) \\
& -F_{c}\left(\left(q_{3}^{*} l\right) * l\right)(y) .
\end{aligned}
$$

It shows that

$$
f(x)=p(x)-(*(q, h, k))(x)-(q * l)+(l * p)(x)-\left(*(q, h, k) *{ }_{1} l\right)(x)-((q * l) * l)(x) .
$$

Similarly, from the generalized convolutions (1.15), (1.19), we have

$$
\begin{aligned}
\Delta_{2} & =\left|\begin{array}{cc}
1 & \left(F_{c} p\right)(y) \\
\left(F_{s} \xi\right)(y)+\sin y\left(F_{c} \eta\right)(y) & \left(F_{s} q\right)(y)
\end{array}\right| \\
& =\left(F_{s} q\right)(y)-F_{s}(\underset{2}{*} p)(y)-F_{s}(\underset{2}{\stackrel{\gamma}{*} p})(y) .
\end{aligned}
$$


Using formulas (3.25), (3.30), we have

$$
\begin{aligned}
& \left(F_{s} g\right)(y)=\left[1+\left(F_{c} l\right)(y)\right]\left[\left(F_{s} q\right)(y)-F_{s}(\xi \underset{2}{\xi *} p)(y)-F_{s}(\eta \underset{2}{r} p)(y)\right] \\
& =\left(F_{s} q\right)(y)-F_{s}(\xi \underset{2}{\xi} p)(y)-F_{s}(\eta \underset{2}{*} p)(y)+F_{s}(\underset{2}{q *} l)-F_{s}((\underset{2}{\xi * p}) * 2)(y) \\
& -F_{s}\left(\left(\begin{array}{c}
\gamma \underset{2}{*} p \\
*
\end{array}\right) * l\right)(y) \text {. }
\end{aligned}
$$

It shows that

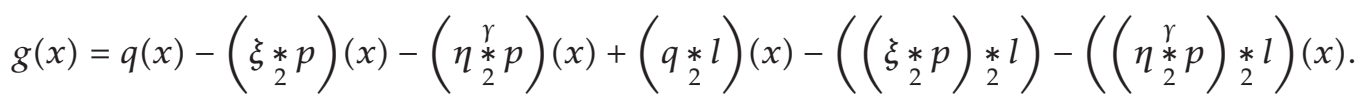

Pair $(f, g)$ defined by fomulae (3.29) and (3.32) is a solution in closed form in $L_{1}\left(\mathbb{R}_{+}\right) \times L_{1}\left(\mathbb{R}_{+}\right)$ of system (3.17). The proof is complete.

\section{Acknowledgments}

In the memory of professor V. A. Kakichev, the author wish to express his deep thanks to him for all his encouragement to the author in this investigative direction. This research is supported partially by Vietnam's National Foundation for Science and Technology Development, Grant no. 101.01.21.09.

\section{References}

[1] I. N. Sneddon, Fourier Transform, McGraw-Hill, New York, NY, USA, 1951.

[2] S. B. Yakubovich, "On the convolution for the Kontorovich-Lebedev transformation and its applications to integral equations," Doklady Akademii Nauk BSSR, vol. 31, no. 2, pp. 101-103, 1987 (Russian).

[3] I. N. Sneddon, The Use of Integral Transforms, McGraw-Hill, New York, NY, USA, 1972.

[4] A. Ardelyi, W. Magnus, F. Oberhettinger, and F. G. Tricomi, Higher Transcendental Function, vol. 1, 2, 3, McGraw-Hill, New York, NY, USA, 1953.

[5] V. A. Kakichev, "On the convolution for integral transforms," Izvestiya Vysshikh Uchebnykh Zavedenii. Matematika, no. 2, pp. 53-62, 1967 (Russian).

[6] N. X. Thao and N. T. Hai, Convolution for Integral Transform and Their Application, Russian Academy, Moscow, Russia, 1997.

[7] N. X. Thao, V. A. Kakichev, and V. K. Tuan, "On the generalized convolutions for Fourier cosine and sine transforms," East-West Journal of Mathematics, vol. 1, no. 1, pp. 85-90, 1998.

[8] N. X. Thao, V. K. Tuan, and N. M. Khoa, "A generalized convolution with a weight function for the Fourier cosine and sine transforms," Fractional Calculus $\mathcal{E}$ Applied Analysis, vol. 7, no. 3, pp. 323-337, 2004.

[9] N. X. Thao and N. M. Khoa, "On the generalized convolution with a weight function for the Fourier sine and cosine transforms," Integral Transforms and Special Functions, vol. 17, no. 9, pp. 673-685, 2006.

[10] S. B. Yakubovich and L. E. Britvina, "Convolutions related to the Fourier and Kontorovich-Lebedev transforms revisited," Integral Transforms and Special Functions, vol. 21, no. 4, pp. 259-276, 2010.

[11] V. A. Kakichev, Polyconvolution, TPTU, Taganrog, Russia, 1997. 
[12] N. X. Thao, "On the polyconvolution for integral transforms," Vestnik Novgorodskogo Gosudarstvennogo Universiteta. Seriya Estestvennye i Tekhnicheskie Nauki, vol. 10, pp. 104-110, 1999.

[13] N. X. Thao and N. D. Hau, "On the polyconvolution for the Fourier cosine and Fourier sine transforms," Acta Mathematica Vietnamica, vol. 33, no. 2, pp. 107-122, 2008.

[14] J. J. Betancor and B. J. González, "Spaces of $L_{p}$-type and the Hankel convolution," Proceedings of the American Mathematical Society, vol. 129, no. 1, pp. 219-228, 2001.

[15] J. J. Betancor, C. Jerez, S. M. Molina, and L. Rodríguez-Mesa, “Distributional convolutors for Fourier transform," Journal of Mathematical Analysis and Applications, vol. 325, no. 1, pp. 459-468, 2007.

[16] J. J. Betancor, M. Linares, and J. M. R. Méndez, "A distributional convolution for a generalized finite Fourier transformation," Proceedings of the American Mathematical Society, vol. 128, no. 2, pp. 547-556, 2000.

[17] H. M. Srivastava and V. K. Tuan, "A new convolution theorem for the stieltjes transform and its application to a class of singular integral equations," Archiv der Mathematik, vol. 64, no. 2, pp. 144 $149,1995$.

[18] H. M. Titchmarsh, Introduction to the Theory of Fourier Integrals, Clarendon Press, Oxford, UK, 2nd edition, 1967

[19] F. G. Tricomi, “On the finite Hilbert transformation," The Quarterly Journal of Mathematics, vol. 2, pp. 199-211, 1951.

[20] V. K. Tuan and M. Saigo, "Convolution of Hankel transform and its application to an integral involving Bessel functions of first kind," International Journal of Mathematics and Mathematical Sciences, vol. 18, no. 3, pp. 545-549, 1995.

[21] S. B. Yakubovich and A. I. Moshinski1̌, "Integral equations and convolutions associated with transformations of Kontorovich-Lebedev type," Differentsial'nye Uravnenia, vol. 29, no. 7, pp. 12721284, 1993.

[22] H. H. Kagiwada and R. Kalaba, Integral Equations via Imbedding Methods, vol. 6 of Applied Mathematics and Computation, Addison-Wesley, London, UK, 1974.

[23] M. G. Kreॉn, "On a new method of solution of linear integral equations of first and second kinds," Doklady Akademii Nauk SSSR, vol. 100, pp. 413-416, 1955 (Russian).

[24] J. N. Tsitsiklis and B. C. Levy, "Integral equations and resolvents of Toeplitz plus Hankel kernels," Tech. Rep. LIDS-P 1170, Laboratory for Information and Decision Systems, Massachusetts Institute of Technology, Cambridge, Mass, USA, 1981.

[25] N. I. Achiezer, Lectures on Approximation Theory, Science Publishing House, Moscow, Russia, 1965. 


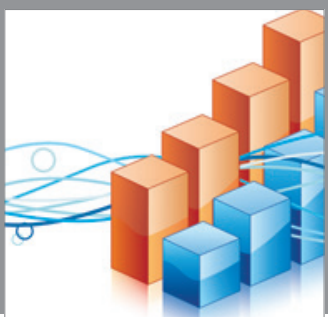

Advances in

Operations Research

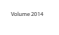

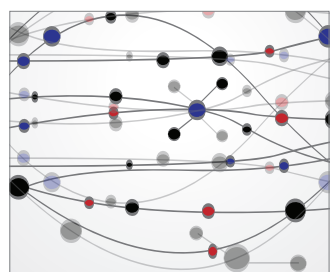

\section{The Scientific} World Journal
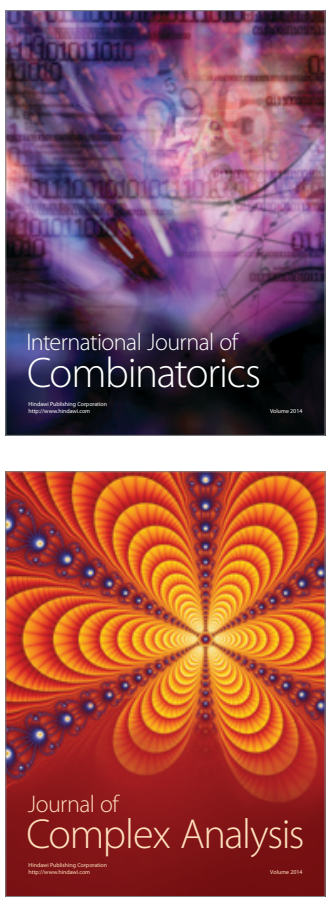

International Journal of

Mathematics and

Mathematical

Sciences
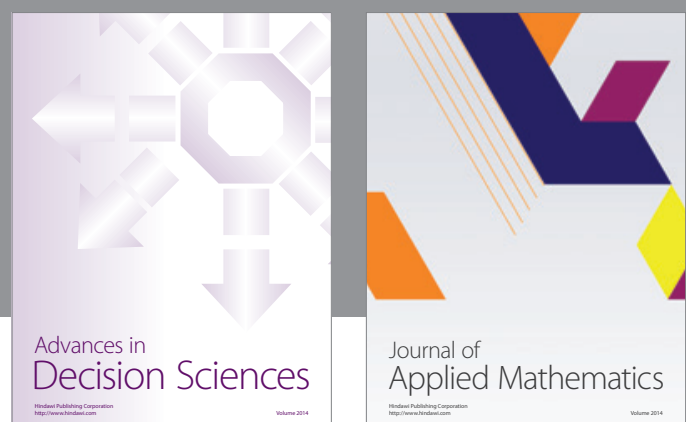

Journal of

Applied Mathematics
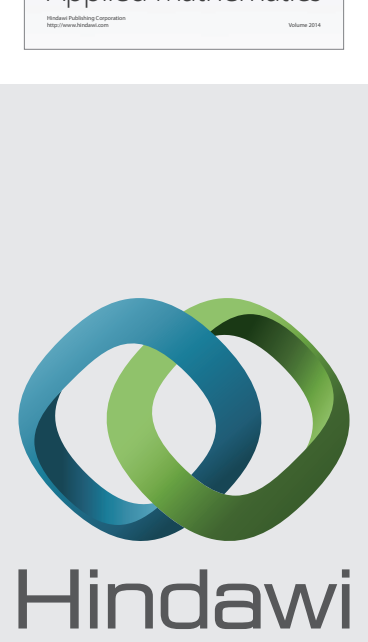

Submit your manuscripts at http://www.hindawi.com
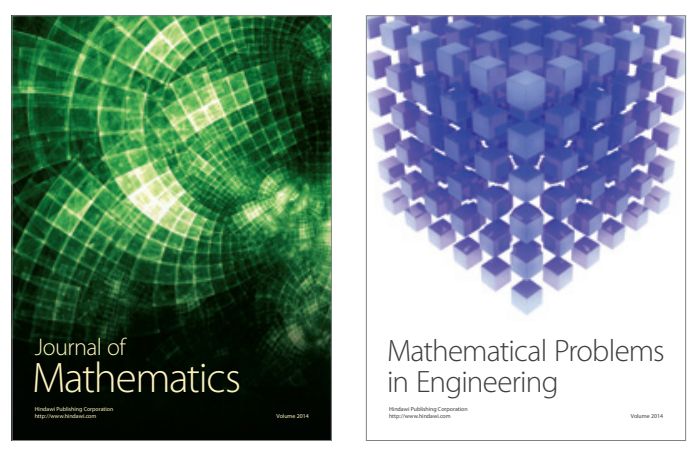

Mathematical Problems in Engineering
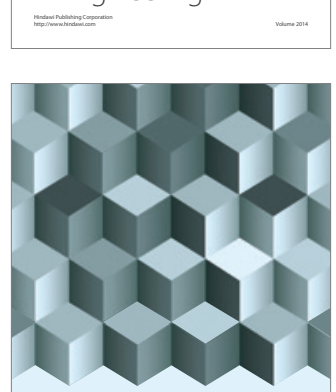

Journal of

Function Spaces
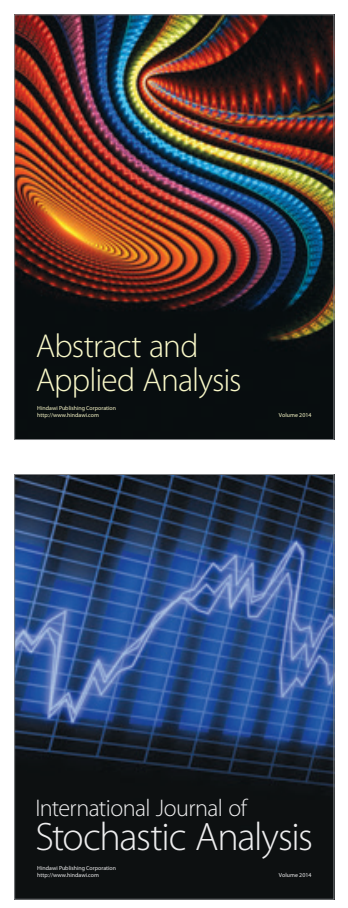

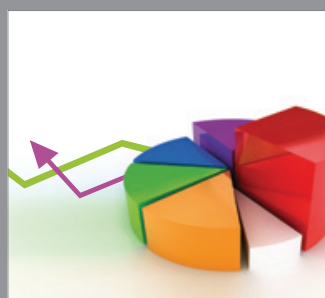

ournal of

Probability and Statistics

Promensencen
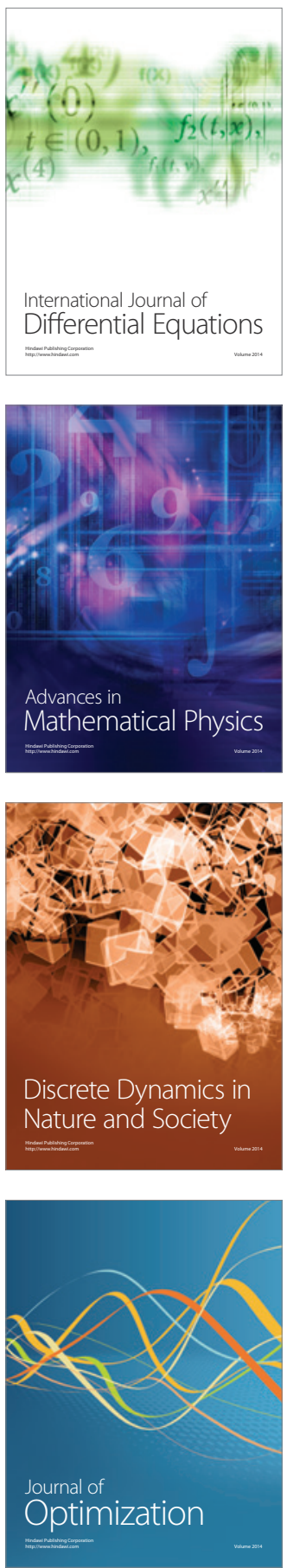